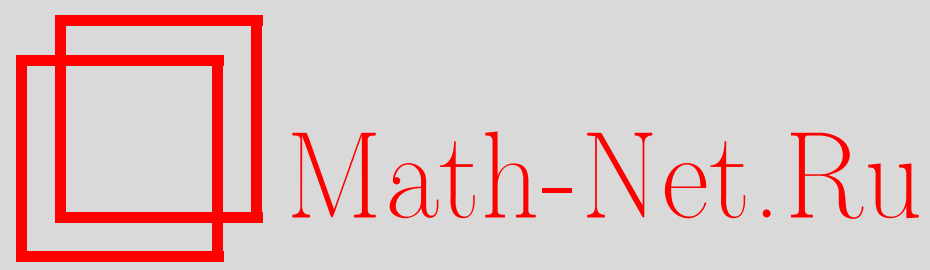

Е. А. Уланский, Тождества для обобщенных полилогарифмов, Матем. заметки, 2003, том 73, выпуск 4, 613-624

DOI: https://doi.org/10.4213/mzm209

Использование Общероссийского математического портала Math-Net.Ru подразумевает, что вы прочитали и согласны с пользовательским соглашением http://www. mathnet.ru/rus/agreement

Параметры загрузки:

IP: 54.197 .217 .227

26 апреля 2023 г., 17:58:35

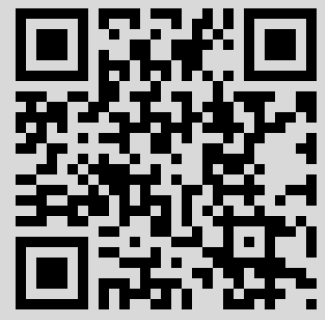




\section{ТОЖДЕСТВА ДЛЯ ОБОБЩЕННЫХ ПОЛИЛОГАРИФМОВ}

\section{Е. А. Уланский}

Изучается поведение обобщенного полилогарифма под действием группы дробнолинейных преобразований аргумента, образованной преобразованиями $z \mapsto 1-z$ и $z \mapsto-z /(1-z)$, что позволяет получать тождества вида

$$
\operatorname{Li}_{k}\left(\frac{-z}{1-z}\right)=-\sum_{|\bar{s}|=k} \operatorname{Li}_{\bar{s}}(z)
$$

Как следствие этих тождеств доказывается линейная независимость обобщенных полилогарифмов и алгебраическая независимость классических полилогарифммов над полем $\mathbb{C}(z)$.

Библиография: 7 названий.

1. Введение. Пусть $\bar{s}=\left(s_{1}, s_{2}, \ldots, s_{k}\right)$-набор с $s_{j} \in \mathbb{N}$. Будем называть величины $|\bar{s}|=s_{1}+s_{2}+\cdots+s_{k}$ и $l(\bar{s})=k$ весом и длиной набора $\bar{s}$ соответственно.

Рассмотрим ряд

$$
\operatorname{Li}_{\left(s_{1}, s_{2}, \ldots, s_{k}\right)}(z):=\sum_{n_{1}>n_{2}>\cdots>n_{k} \geqslant 1} \frac{z^{n_{1}}}{n_{1}^{s_{1}} n_{2}^{s_{2}} \cdots n_{k}^{s_{k}}}
$$

который сходится в области $|z|<1$ и назьвается обобщенным полилогарифмом. Функции, определяемые такими рядами, удовлетворяют системе дифференциальньх уравнений

$$
\begin{aligned}
\frac{d}{d z} \operatorname{Li}_{\left(s_{1}, s_{2}, \ldots, s_{n}\right)}(z)=\frac{1}{z} \operatorname{Li}_{\left(s_{1}-1, s_{2}, \ldots, s_{n}\right)}(z), & \text { если } s_{1}>1, \\
\frac{d}{d z} \operatorname{Li}_{\left(1, s_{2}, \ldots, s_{n}\right)}(z)=\frac{1}{1-z} \operatorname{Li}_{\left(s_{2}, \ldots, s_{n}\right)}(z) & \text { иначе. }
\end{aligned}
$$

Пусть $\bar{s}=\left(s_{1}, s_{2}, \ldots, s_{k}\right) \in \mathbb{C}^{k}$ и $\operatorname{Re} s_{j}>0$. Ряд

$$
\zeta(\bar{s}):=\sum_{n_{1}>n_{2}>\cdots>n_{k} \geqslant 1} \frac{1}{n_{1}^{s_{1}} n_{2}^{s_{2}} \cdots n_{k}^{s_{k}}}
$$

назьвается кратной дзета-функиией. Он сходится при $\operatorname{Re} s_{1}>1$. В случае, когда $k=1$, мы получаем классическую дзета-функцию Римана. Если же $s_{1}, s_{2}, \ldots, s_{k} \in \mathbb{N}$, $s_{1} \geqslant 2$, то имеет место равенство $\zeta(\bar{s})=\operatorname{Li}_{\bar{s}}(1)$. 
Иног да удобнее использовать в качестве индексов полилогарифмов не наборы натуральных чисел, а слова алфавита, состоящего из двух букв $\left\{x_{0}, x_{1}\right\}$. Пусть $X^{*}$ есть множество всех слов, состоящих из букв $x_{0}$ и $x_{1}$, содержащее в том числе и пустое слово. Для любого слова $w$ множества $X^{*}$ определен вес $|w|$, которьй равен количеству всех букв в этом слове, и длина $l(w)$, равная количеству букв $x_{1}$, встречающихся в этом слове. В частности, если $|w|=l(w)=k$, то $w=x_{1}^{k}$. Для пустого слова полагается $|\varnothing|=l(\varnothing)=0$.

Любому натуральному набору $\bar{s}=\left(s_{1}, \ldots, s_{k}\right)$ сопоставим слово

$$
w=x_{0}^{s_{1}-1} x_{1} x_{0}^{s_{2}-1} x_{1} \ldots x_{0}^{s_{k}-1} x_{1} .
$$

Таким образом, каждому полилогарифму вида (1) поставлено в соответствие некоторое слово из $X^{*} x_{1}$, причем понятия длины и веса согласованы. При этом каждому слову из $X^{*} x_{1}$ соответствует некоторый полилогарифм, так как оно единственным образом представляется в виде (3). Аналогично, для любого слова $w \in x_{0} X^{*} x_{1}$ определяется кратная дзета-функция $\zeta(w)$.

Обобщенньй полилогарифм можно определить для произвольного слова $w$ из $X^{*}$ следуюшим образом. Положим $\operatorname{Li}_{\varnothing}(z)=1$ и

$$
\begin{aligned}
& \operatorname{Li}_{w}(z)= \begin{cases}\frac{\ln ^{j} z}{j !}, & \text { если } w=x_{0}^{j}, j \geqslant 1, \\
\int_{0}^{z} \omega_{i}(t) \operatorname{Li}_{u}(t) d t, & \text { если } w=x_{i} u \text { содержит букву } x_{1},\end{cases} \\
& \omega_{0}(z)=\frac{1}{z}, \quad \omega_{1}(z)=\frac{1}{1-z} .
\end{aligned}
$$

Из этого определения легко вьвести следующую формулу для обобщенного полилогарифмма.

Для любого целого неотрицательного числа $k$ определим множество слов $X_{k}$, полагая $X_{0}=\{\varnothing\}$ и $X_{k}=\left\{w \in X^{*} x_{1}: l(w)=k\right\}$ при $k \geqslant 1$. Далее, произвольное слово $w$ из $X^{*}$ запишем в виде

$$
w=x_{1}^{s_{1}} x_{0}^{i_{1}} x_{1}^{s_{2}+1} x_{0}^{i_{2}} \ldots x_{1}^{s_{q-1}+1} x_{0}^{i_{q-1}} x_{1}^{s_{q}+1} x_{0}^{i_{q}}
$$

где $q \geqslant 1, s_{1} \geqslant 0, \ldots, s_{q} \geqslant 0, i_{1} \geqslant 1, \ldots, i_{q-1} \geqslant 1, i_{q} \geqslant 0$. Обозначим через $C_{m}^{n}$ биномиальные коэффициенты:

$$
C_{m}^{n}=\frac{m !}{(m-n) ! n !} .
$$

ПРЕДЛОЖЕНИЕ 1. В приведенных обозначениях имеет место разложсение

$$
\begin{aligned}
\operatorname{Li}_{w}(z)= & \sum_{0 \leqslant j_{1}+\cdots+j_{q} \leqslant i_{q}} C_{i_{1}+j_{1}}^{j_{1}} \cdots C_{i_{q-1}+j_{q-1}}^{j_{q-1}} \\
& \times \sum_{\substack{\left|p_{1}\right|+\cdots+\left|p_{q}\right|=s_{1}+\cdots+s_{q}+i_{q}-j_{1}-\cdots-j_{q} \\
p_{1} \in X_{s_{1}}, \ldots, p_{q} \in X_{s_{q}}}}(-1)^{i_{q}-j_{q}} \frac{\ln ^{j_{q}}(z)}{j_{q} !} \\
& \quad \times \operatorname{Li}_{p_{1} x_{0}^{i_{1}+j_{1}} x_{1} \ldots p_{q-1} x_{0}^{i_{q-1}+j_{q-1}} x_{1} p_{q}}(z) .
\end{aligned}
$$


ЗАмЕчАНИЕ. Нетрудно заметить, что в этой формуле использованы только обобщенные полилогарифмы, отвечающие либо пустому слову, либо словам из $X^{*} x_{1}$, которые были определены выше. Более того, имеют место следуюшие свойства:

1) если в правой части (5) присутствует слагаемоевида $\ln ^{j} z \operatorname{Li}_{v}(z)$, то $|v|=|w|-j$;

2 ) если $w=v x_{1} x_{0}^{k}$, то в правую часть (5) функция $\ln z$ входит в степени не выше $k$;

3 ) если $w$ начинается с $x_{0}$, то существует число $\mathrm{Li}_{w}(1)$, а в случае, когда $w$ содержит букву $x_{1}$, имеет место равенство $\mathrm{Li}_{w}(0)=0$.

ДоКАЗАТЕЛЬСТво. Проверяется формула (5) с помощью следующих равенств:

$$
\begin{aligned}
\int \frac{\ln ^{m}(z)}{m !} \frac{1}{1-z} \operatorname{Li}_{w}(z) d z & =\sum_{i=0}^{m}(-1)^{i} \frac{\ln ^{m-i}(z)}{(m-i) !} \operatorname{Li}_{x_{0}^{i} x_{1} w}(z)+C, \quad w \in X^{*} x_{1} \cup \varnothing \\
\int \frac{\ln ^{m}(z)}{m !} \frac{1}{z} \operatorname{Li}_{w}(z) d z & =\sum_{i=0}^{m}(-1)^{i} \frac{\ln ^{m-i}(z)}{(m-i) !} \operatorname{Li}_{x_{0}^{i+1} w}(z)+C, \quad w \in X^{*} x_{1}
\end{aligned}
$$

здесь $m$ - целое неотрицательное число. Кроме того,

$$
\sum_{i=0}^{k} C_{s+i}^{i}=C_{s+1+k}^{k}, \quad s \geqslant 0, \quad k \geqslant 0
$$

и

$$
\left\{w:|w|=s+1+k, w \in X_{s+1}\right\}=\bigcup_{i=0}^{k}\left\{x_{0}^{i} x_{1} v:|v|=s+k-i, v \in X_{s}\right\}, \quad s \geqslant 0, \quad k \geqslant 0,
$$

где объединяемые множества попарно не пересекаются. Это завершает проверку разложения (5).

Из определения следует, что обобщенные полилогарифмы удовлетворяют системе дифференциальных уравнений

$$
\frac{d}{d z} \operatorname{Li}_{x_{0} w}(z)=\frac{1}{z} \operatorname{Li}_{w}(z), \quad \frac{d}{d z} \operatorname{Li}_{x_{1} w}(z)=\frac{1}{1-z} \operatorname{Li}_{w}(z)
$$

для любого слова $w \in X^{*}$. При этом для слов из $X^{*} x_{1}$ система (6) согласована с системой (2).

В статье [1] изучается поведение функций $\mathrm{Li}_{\left(s_{1}, \ldots s_{k}\right)}(z)$ под действием групшы дробно-линейных преобразований

$$
z, \quad 1-z, \frac{-z}{1-z}, \frac{1}{z}, \frac{1}{1-z}, \frac{z-1}{z} .
$$

Метод, предложенньй в [1], эффективен и позволил при помощи ЭВМ и системы формальных вычислений АХIOМ выразить в некоторых случаях при $k \leqslant 3$ и $s_{1}+\cdots+s_{k} \leqslant 4$ значения обобщенных полилогарифмов в точках $1-z, 1 / z,(z-1) / z$ через значения таких же функций в точке $z$, а также в терминах $\ln z$ и чисел $\pi, \zeta(m)$.

В настоящей работе, выбирая в качестве образующих групы преобразования $-z /(1-z)$ и $1-z$, мы предлагаем явные формулы в общем случае. 
ТЕоремА 1. Для любых иелых неотрицательных чисел $s_{1}, \ldots, s_{n}$ и комплексного числа z с условиями $|z|<1,|z /(1-z)|<1$ выполняется равенство

$$
\mathrm{Li}_{x_{0}^{s_{1}} x_{1} \ldots x_{0}^{s_{n}} x_{1}}\left(\frac{-z}{1-z}\right)=(-1)^{n} \sum_{\left|p_{1}\right|=s_{1}, \ldots,\left|p_{n}\right|=s_{n}} \operatorname{Li}_{p_{1} x_{1} \ldots p_{n} x_{1}}(z) .
$$

СЛЕДСТВИЕ. Пусть $n=1 u s_{1}=k-1$. Тогда

$$
\operatorname{Li}_{x_{0}^{k-1} x_{1}}\left(\frac{-z}{1-z}\right)=-\sum_{|p|=k-1} \operatorname{Li}_{p x_{1}}(z)
$$

Полученное равенство эквивалентно тождеству, помещенному в аннотации.

Зададим на множестве слов $X^{*}$ отображение $\sigma$, которое меняет буквы $x_{0}$ и $x_{1}$ между собой. Например, $\sigma(\varnothing)=\varnothing$, а $\sigma\left(x_{0}^{3} x_{1}\right)=x_{1}^{3} x_{0}$.

ТЕОРема 2. Пусть имеется слово $w$ из $X^{*}$ и комплексное число $z$ с условиями $|z|<1,|1-z|<1$. Тогда

$$
\operatorname{Li}_{w}(1-z)=\sum_{u v=w}(-1)^{|u|} D_{v} \operatorname{Li}_{\sigma(u)}(z)
$$

где действительные постоянные $D_{v}$ определяются следующими правилами: $D_{\varnothing}=1 ; D_{v}=\mathrm{Li}_{v}(1)$, если $v$ начинается $с x_{0}, u$

$$
D_{v}=-\sum_{\substack{p t=v \\|p|>0}}(-1)^{|p|} D_{t} \operatorname{Li}_{\sigma(p)}(1)
$$

если $v$ начинается $c x_{1}$.

В п. 4 и п. 5 данной статьи с помощю теоремы 2 доказьвается линейная независимость обобщенных полилогарифмов вида (1) и алгебраическая независимость классических полилогарифмов над полем $\mathbb{C}(z)$.

\section{2. Доказательства теорем 1 и 2.}

ДокАЗАТЕЛЬСтво ТЕОремЫ 1 . Согласно (6) для всех $w \in X^{*} x_{1}$ имеет место система

$$
\frac{d}{d z} \operatorname{Li}_{x_{0} w}\left(\frac{-z}{1-z}\right)=\frac{1}{z(1-z)} \operatorname{Li}_{w}\left(\frac{-z}{1-z}\right), \quad \frac{d}{d z} \operatorname{Li}_{x_{1} w}\left(\frac{-z}{1-z}\right)=\frac{-1}{1-z} \operatorname{Li}_{w}\left(\frac{-z}{1-z}\right) .
$$

Теперь заметим, что для любого слова $w \in X^{*} x_{1}$ вьполнено $\operatorname{Li}_{w}(0)=0$ и

$$
\frac{d}{d z}\left(\operatorname{Li}_{x_{0} w}(z)+\operatorname{Li}_{x_{1} w}(z)\right)=\frac{1}{z(1-z)} \operatorname{Li}_{w}(z) .
$$

Поэтому если $f(0)=0$ и $f^{\prime}(z)=\operatorname{Li}_{w}(z) /(z(1-z))$, то

$$
f(z)=\mathrm{Li}_{x_{0} w}(z)+\mathrm{Li}_{x_{1} w}(z) .
$$


Если же $f(0)=0$ и $f^{\prime}(z)=\operatorname{Li}_{w}(z) /(1-z)$, то

$$
f(z)=\operatorname{Li}_{x_{1} w}(z) .
$$

Положим $w=x_{0}^{s_{1}} x_{1} \ldots x_{0}^{s_{n}} x_{1}$ и докажем утверждение теоремы индукцией по $|w|$. При $|w|=1$ имеется единственное слово $w=x_{1}$, поэтому необходимо проверить равенство

$$
\operatorname{Li}_{x_{1}}\left(\frac{-z}{1-z}\right)=-\operatorname{Li}_{x_{1}}(z)
$$

Но из определения следует, что

$$
\operatorname{Li}_{x_{1}}(z)=\sum_{n \geqslant 1} \frac{z^{n}}{n}=-\ln (1-z)
$$

отсюда

$$
\operatorname{Li}_{x_{1}}\left(\frac{-z}{1-z}\right)=-\ln \left(1+\frac{z}{1-z}\right)=-\ln \frac{1}{1-z}=\ln (1-z)=-\operatorname{Li}_{x_{1}}(z) .
$$

Пусть, далее, $|w| \geqslant 1$ и для $w$ теорема уже доказана. Требуется установить, что теорема верна для слов $x_{0} w$ и $x_{1} w$. Для слова $x_{0} w$ согласно (7) получаем

$$
\frac{d}{d z} \operatorname{Li}_{x_{0} w}\left(\frac{-z}{1-z}\right)=\frac{1}{z(1-z)} \operatorname{Li}_{w}\left(\frac{-z}{1-z}\right)=\frac{(-1)^{n}}{z(1-z)} \sum_{\left|p_{1}\right|=s_{1}, \ldots,\left|p_{n}\right|=s_{n}} \operatorname{Li}_{p_{1} x_{1} \ldots p_{n} x_{1}}(z)
$$

Применяя (8), находим

$$
\begin{aligned}
\operatorname{Li}_{x_{0} w}\left(\frac{-z}{1-z}\right) & =(-1)^{n} \sum_{\left|p_{1}\right|=s_{1}, \ldots,\left|p_{n}\right|=s_{n}}\left(\operatorname{Li}_{x_{0} p_{1} x_{1} \ldots p_{n} x_{1}}(z)+\operatorname{Li}_{x_{1} p_{1} x_{1} \ldots p_{n} x_{1}}(z)\right) \\
& =(-1)^{n} \sum_{\left|p_{1}\right|=s_{1}+1, \ldots,\left|p_{n}\right|=s_{n}} \operatorname{Li}_{p_{1} x_{1} \ldots p_{n} x_{1}}(z) .
\end{aligned}
$$

Для слова $x_{1} w$ согласно (7)

$$
\frac{d}{d z} \operatorname{Li}_{x_{1} w}\left(\frac{-z}{1-z}\right)=\frac{-1}{1-z} \operatorname{Li}_{w}\left(\frac{-z}{1-z}\right)=\frac{(-1)^{n+1}}{1-z} \sum_{\left|p_{1}\right|=s_{1}, \ldots,\left|p_{n}\right|=s_{n}} \operatorname{Li}_{p_{1} x_{1} \ldots p_{n} x_{1}}(z)
$$

а использование правила (9) дает

$$
\operatorname{Li}_{x_{1} w}\left(\frac{-z}{1-z}\right)=(-1)^{n+1} \sum_{\left|p_{1}\right|=s_{1}, \ldots,\left|p_{n}\right|=s_{n}} \operatorname{Li}_{x_{1} p_{1} x_{1} \ldots p_{n} x_{1}}(z)
$$

что в точности совпадает с утверждением теоремы.

Теорема 1 полностью доказана. 
ДоКАЗАТЕЛЬСТВо ТЕОРЕМЫ 2 . Из (6) получаем, что для любого слова $w \in X^{*}$ верно

$$
\frac{d}{d z} \operatorname{Li}_{x_{0} w}(1-z)=\frac{-1}{1-z} \operatorname{Li}_{w}(1-z), \quad \frac{d}{d z} \operatorname{Li}_{x_{1} w}(1-z)=\frac{-1}{z} \operatorname{Li}_{w}(1-z) .
$$

Проведем доказательство теоремы индукцией по $|w|$. При $|w|=0$ имеется только пустое слово и требуется проверить очевидное тождество $\mathrm{Li}_{\varnothing}(1-z)=\mathrm{Li}_{\varnothing}(z)$.

Пусть $|w| \geqslant 0$ и для слова $w$ теорема верна. Докажем, что теорема также справедлива для слов $x_{0} w$ и $x_{1} w$. Мы рассмотрим только второй из этих случаев; первый рассматривается аналогично. Пользуясь (10) и индукционньм предположением, получаем

$$
\frac{d}{d z} \operatorname{Li}_{x_{1} w}(1-z)=\frac{-1}{z} \operatorname{Li}_{w}(1-z)=\frac{1}{z} \sum_{u v=w}(-1)^{|u|+1} D_{v} \operatorname{Li}_{\sigma(u)}(z) .
$$

Благодаря (6), находим

$$
\operatorname{Li}_{x_{1} w}(1-z)=\sum_{u v=w}(-1)^{|u|+1} D_{v} \operatorname{Li}_{x_{0} \sigma(u)}(z)+C .
$$

Обозначим полученную константу интегрирования через $D_{x_{1} w}$; тогда

$$
\operatorname{Li}_{x_{1} w}(1-z)=\sum_{u v=x_{1} w}(-1)^{|u|} D_{v} \operatorname{Li}_{\sigma(u)}(z) .
$$

Найдем константу $D_{x_{1} w}$, положив $z=1$. Получим

$$
D_{x_{1} w}=-\sum_{\substack{u v=x_{1} w \\|u|>0}}(-1)^{|u|} D_{v} \operatorname{Li}_{\sigma(u)}(1)
$$

что и требовалось.

При доказательстве теоремы для слова $x_{0} w$ константа интегрирования находится подстановкой $z=0$.

Теорема 2 полностью доказана.

ЗАмЕчАнИЕ. Пусть $v$ - пустое слово или произвольное из $X^{*} x_{1}$. Тогда в разложении (5) функции $\mathrm{Li}_{v x_{0}^{j}}(z)$ при $\ln ^{j}(z)$ имеется единственное слагаемое

$$
\frac{1}{j !} \mathrm{Li}_{v}(z)
$$

В соответствии с этим из теоремы 2 заключаем, что в выражении для $\mathrm{Li}_{\sigma(v) x_{1}^{j}}(1-z)$ при $\ln ^{j}(z)$ присутствуют следуюшие слагаемые:

$$
\frac{(-1)^{|v|+j}}{j !} \mathrm{Li}_{v}(z)+\sum_{|u|<|v|} C_{u} \operatorname{Li}_{u}(z)
$$

где $C_{u}$ - некоторые действительные константы.

Если в условии теоремы 2 взять слово вида (4) с $s_{1}=0$ или $1, s_{2}=\cdots=s_{q}=0$ и $i_{1}=\cdots=i_{q-1}=1, i_{q}=0$, то получим 
СЛЕДСТВИЕ. Для любого $n=0,1,2, \ldots$ имеют место равенства

$$
\begin{aligned}
\operatorname{Li}_{\{2\}_{n}}(1-z)= & \sum_{k=1}^{n} \gamma_{n-k} \cdot\left(\ln z \operatorname{Li}_{\left(1,\{2\}_{k-1}\right)}(z)-\operatorname{Li}_{\{2\}_{k}}(z)\right. \\
& \left.-2 \sum_{\substack{|\bar{s}|=k \\
l(\bar{s})=k-1}} \operatorname{Li}_{\left(1, s_{1}+1, \ldots, s_{k-1}+1\right)}(z)\right) \\
& +2 \sum_{k=1}^{n-1} \beta_{k} \cdot \operatorname{Li}_{\left(1,\{2\}_{n-1-k}\right)}(z)+\gamma_{n}, \\
\operatorname{Li}_{\left(1,\{2\}_{n}\right)}(1-z)= & \sum_{k=0}^{n} \gamma_{n-k} \cdot\left(-\ln z \operatorname{Li}_{\{2\}_{k}}(z)+2 \sum_{\substack{|\bar{s}|=k+1 \\
l(\bar{s})=k}} \operatorname{Li}_{\left(s_{1}+1, \ldots, s_{k}+1\right)}(z)\right) \\
& -2 \sum_{k=1}^{n} \beta_{k} \cdot \operatorname{Li}_{\{2\}_{n-k}}(z),
\end{aligned}
$$

əде $\gamma_{k}=\zeta\left(\{2\}_{k}\right), k=0,1,2, \ldots, u$

$$
\beta_{k}=\sum_{\substack{|\bar{s}|=k+1 \\ l(\bar{s})=k}} \zeta\left(s_{1}+1, \ldots, s_{k}+1\right), \quad k=1,2, \ldots
$$

(Здесь было использовано определение обобщенного полилогарифма (1) и обозначение

$$
\{t\}_{k}=(\underbrace{t, \ldots, t}_{k \text { раз }})
$$

при $k=1,2, \ldots$ и $\left.\{t\}_{0}=\varnothing.\right)$

Этот результат уточняет лемму 13 из статьи [2].

3. Некоторые замечания. Иногда вместо $\mathrm{Li}_{\left(s_{1}, \ldots, s_{k}\right)}(z)$ удобнее рассматривать функции, которые мы обозначим

$$
\operatorname{Le}_{\left(s_{1}, \ldots, s_{k}\right)}(z)=\sum_{n_{1} \geqslant n_{2} \geqslant \cdots \geqslant n_{k} \geqslant 1} \frac{z^{n_{1}}}{n_{1}^{s_{1}} n_{2}^{s_{2}} \cdots n_{k}^{s_{k}}} .
$$

Будем называть такой ряд нестрогим обобщенным полилогарифмом.

Нет необходимости отдельно для таких рядов доказывать теоремы 1 и 2 , поскольку справедливо

ПРЕДЛОЖЕНИЕ 2. Имеет место равенство

$$
\operatorname{Le}_{\left(s_{1}, \ldots, s_{k}\right)}(z)=\sum_{\bar{p}} \operatorname{Li}_{\bar{p}}(z)
$$

где $\bar{p}$ пробегает все наборы вида $\left(s_{1} * s_{2} * \cdots * s_{k}\right)$. Здесь '*' можсет быть либо знаком '+', либо знаком ','; всего таких наборов $2^{k-1}$.

В несколько других терминах для случая $z=1$ это утверждение было сформулировано в статье [3]. Для доказательства достаточно установить, что верна 
ЛЕмма 1. В обозначениях предложсения 2 выполняется следующее равенство:

$$
\sum_{n_{1}>\cdots>n_{l} \geqslant n_{l+1} \geqslant \cdots \geqslant n_{k} \geqslant 1} \frac{z^{n_{1}}}{n_{1}^{s_{1}} \cdots n_{k}^{s_{k}}}=\sum_{\bar{p}=\left(s_{1}, \ldots, s_{l} * s_{l+1} * \cdots * s_{k}\right)} \operatorname{Li}_{\bar{p}}(z) .
$$

ДокАЗАТЕЛЬСТВО проведем индукцией по $k=l(\bar{s})$. При $k=1$ утверждение очевидно. Пусть $k>1$ и для $k-1$ лемма верна.

Проведем нисходящую индукцию по $l$. При $l=k$ утверждение выполняется тривиально. Допустим, что $1 \leqslant l<k$ и для $l+1$ лемма верна. Тогда

$$
\begin{aligned}
& \sum_{n_{1}>\cdots>n_{l} \geqslant n_{l+1} \geqslant \cdots \geqslant n_{k} \geqslant 1} \frac{z^{n_{1}}}{n_{1}^{s_{1}} \cdots n_{k}^{s_{k}}} \\
& =\sum_{n_{1}>\cdots>n_{l+1} \geqslant n_{l+2} \geqslant \cdots \geqslant n_{k} \geqslant 1} \frac{z^{n_{1}}}{n_{1}^{s_{1}} \cdots n_{k}^{s_{k}}} \\
& +\sum_{n_{1}>\cdots>n_{l} \geqslant n_{l+2} \geqslant \cdots \geqslant n_{k} \geqslant 1} \frac{z^{n_{1}}}{n_{1}^{s_{1}} \cdots n_{l-1}^{s_{l-1}} \cdot n_{l}^{s_{l}+s_{l+1}} \cdot n_{l+2}^{s_{l+2}} \cdots n_{k}^{s_{k}}} \\
& =\sum_{\bar{p}=\left(s_{1}, \ldots, s_{l}, s_{l+1} * s_{l+2} * \cdots * s_{k}\right)} \operatorname{Li}_{\bar{p}}(z)+\sum_{\bar{p}=\left(s_{1}, \ldots, s_{l}+s_{l+1} * s_{l+2} * \cdots * s_{k}\right)} \operatorname{Li}_{\bar{p}}(z) \\
& =\sum_{\bar{p}=\left(s_{1}, \ldots, s_{l} * s_{l+1} * \cdots * s_{k}\right)} \operatorname{Li}_{\bar{p}}(z) \text {. }
\end{aligned}
$$

Здесь мы дважды воспользовались предположением индукции и определением операции '*'. Шаг индукции по $l$ и $k$ совершен. Лемма доказана.

Обратную связь между строгими и нестрогими полилогарифмами устанавливает

ПРЕДЛОЖЕНИЕ 3. Имеет место равенство

$$
\operatorname{Li}_{\left(s_{1}, \ldots, s_{k}\right)}(z)=\sum_{\bar{p}}(-1)^{\alpha(\bar{p})} \operatorname{Le}_{\bar{p}}(z)
$$

где $\bar{p}$ пробегает все наборы вида $\left(s_{1} * s_{2} * \cdots * s_{k}\right)$. Здесь, как и ранее, '*' можсет быть либо знаком '+', либо знаком ','; степень $\alpha(\bar{p})$ равна количеству знаков '+' в наборе $\bar{p}$.

Доказывается предложение 3 аналогично предложению 2.

4. Линейная независимость. Линейная независимость обобщенных полилогарифмов над полем комплексных чисел была доказана в [4], [5]. Мы покажем, что это есть простое следствие теоремы 2.

ТЕОремА 3. Обобщенные полилогарифмы, отвечающие различным словам из $X^{*} x_{1}$ и пустому слову, линейно независимы над полем $\mathbb{C}(z)$. 
ДоКАЗАТЕЛЬСТВО. Достаточно установить, что для любого целого неотрицательного числа $m$ функции $\operatorname{Li}_{w}(z), w \in X^{*} x_{1} \cup\{\varnothing\},|w| \leqslant m$, линейно независимынад $\mathbb{C}(z)$.

Проведем индукцию по $m$. При $m=0$ имеем $\operatorname{Li}_{\varnothing}(z)=1$ и утверждение очевидно.

Пусть $m>0$ и для $m-1$ утверждение верно. Допустим в области $|z|<1$

$$
\sum_{w \in X^{*} x_{1} \cup\{\varnothing\},|w| \leqslant m} \lambda_{w}(z) \operatorname{Li}_{w}(z) \equiv 0, \quad \lambda_{w}(z) \in \mathbb{C}(z),
$$

причем сушествует слово $u \in X^{*} x_{1},|u|=m$, такое, что $\lambda_{u}(z) \not \equiv 0$. Тогда в области $|1-z|<1$

$$
\sum_{w \in X^{*} x_{1} \cup\{\varnothing\},|w| \leqslant m} \lambda_{w}(1-z) \operatorname{Li}_{w}(1-z) \equiv 0 .
$$

Согласно теореме 2 в области $M=\{z:|z|<1,|1-z|<1\}$ вьполнено

$$
0 \equiv \sum_{w \in X^{*} x_{1} \cup\{\varnothing\},|w| \leqslant m} \lambda_{w}(1-z) \sum_{u v=w}(-1)^{|u|} D_{v} \operatorname{Li}_{\sigma(u)}(z)=\sum_{l=0}^{m} B_{l}(z) \ln ^{l} z
$$

где (см. замечание к предложению 1)

$$
B_{l}(z)=\sum_{v \in X^{*} x_{1} \cup\{\varnothing\},|v| \leqslant m-l} \mu_{v}(z) \operatorname{Li}_{v}(z), \quad \mu_{v}(z) \in \mathbb{C}(z) .
$$

Отметим, что множители $B_{l}(z)$ представляют собой однозначные в области $|z|<1$ функции.

Пусть $z_{0} \in M$. Функцию $F(z)=\sum_{l=0}^{m} B_{l}(z) \cdot \ln ^{l}(z)$ можно аналитически продолжить вдоль замкнутого пути $\gamma$ с началом в точке $z_{0}$, представляющего собой окружность $|z|=\left|z_{0}\right|$, и ориентированного в отрищательном направлении. Полученная функция $G(z)$, с одной стороны, тождественно равна нулю, а с другой стороны,

$$
G(z)=\sum_{l=0}^{m} B_{l}(z) \cdot(\ln z+2 \pi i)^{l} .
$$

Таким образом, многочлен

$$
H(x, z)=\sum_{l=0}^{m} B_{l}(z) \cdot x^{l}
$$

степени $m$ по переменной $x$ для любого $z \in M$ имеет бесконечное множество корней $x_{k}=\ln z+2 \pi i k$, где $k \in \mathbb{Z}$. Это означает, что все его коэффициенты $B_{l}(z)$ тождественно равны нулю.

Пусть $j$ - наибольшее натуральное число такое, что найдется $u=v x_{1}^{j}$ с условиями $|u|=m$ и $\lambda_{u}(z) \not \equiv 0$. По нашему предположению оно сушествует. Запишем

$$
B_{j}(z)=\sum_{v \in X^{*} x_{1} \cup\{\varnothing\},|v| \leqslant m-j} \mu_{v}(z) \operatorname{Li}_{v}(z) .
$$

В сумме справа полилогарифмы $\operatorname{Li}_{v}(z)$ с $|v|=m-j$ согласно замечанию к предложению 1 могут присутствовать только в выражениях для $\operatorname{Li}_{p}(1-z)$, где $|p|=m, p=t x_{1}^{k}$, 
$k \geqslant j$. Но при $k>j$ по определению числа $j$ имеем $\lambda_{p} \equiv 0$. Если же $k=j$, то в соответствии с замечанием после доказательства теоремы 2 полилогарифм $\operatorname{Li}_{v}(z) \mathrm{c}|v|=m-j$ присутствует только в выражении для $\mathrm{Li}_{\sigma(v)} x_{1}^{j}(1-z)$. Поэтому соответствующий коэффициент равен

$$
\mu_{v}(z)=\frac{(-1)^{m}}{j !} \lambda_{\sigma(v) x_{1}^{j}}(1-z)
$$

и можно записать

$$
\begin{aligned}
B_{j}(z)= & \frac{(-1)^{m}}{j !} \sum_{v \in X^{*} x_{1} \cup\{\varnothing\},|v|=m-j} \lambda_{\sigma(v) x_{1}^{j}}(1-z) \operatorname{Li}_{v}(z) \\
+\sum_{v \in X^{*} x_{1} \cup\{\varnothing\},|v|<m-j} \mu_{v}(z) \operatorname{Li}_{v}(z) . &
\end{aligned}
$$

Так как $j \geqslant 1$, то $B_{j}(z)$ представляет собой тождественно равную нулю линейную комбинацию полилогарифмов, отвечающих словам $w$ из $X^{*} x_{1} \cup\{\varnothing\},|w| \leqslant m-1$, с коэффициентами из множества рациональньх функций. По предположению индукции все коэффициенты, стоящие при обобщенных полилогарифмах в $B_{j}(z)$, тождественно равны нулю. Следовательно, для всех $u=v x_{1}^{j},|u|=m$, вьполнено $\lambda_{u} \equiv 0$. Это приводит нас к противоречию с определением числа $j$, которое и завершает индукционньй шаг. Теорема доказана.

ЗАмЕчАниЕ. Утверждение теоремы 3 остается в силе, если считать, что $\lambda_{w}-$ мероморфные функции.

5. Алгебраическая независимость. Этот пункт посвящен доказательству алгебраической независимости над $\mathbb{C}(z)$ классических полилогарифмов

$$
\operatorname{Li}_{k}(z)=\sum_{n=1}^{\infty} \frac{z^{n}}{n^{k}}, \quad k=1,2, \ldots
$$

Этот факт тривиально следует из одной общей леммы, доказанной в 1961г. А. Б. Шидловским [6] (см. также [7, §3]); другое доказательство опубликовано в работе [5].

ЛЕмма 2. Для любого натурального числа $k$ и иелых неотрицательных чисел $j_{1}, \ldots, j_{k}$ справедливо равенство

$$
\operatorname{Li}_{1}^{j_{1}}(z) \operatorname{Li}_{2}^{j_{2}}(z) \cdots \operatorname{Li}_{k}^{j_{k}}(z)=\sum_{\substack{|\bar{r}|=j_{1}+2 j_{2}+\cdots+k j_{k} \\ l(\bar{r})=j_{1}+j_{2}+\cdots+j_{k}}} C_{\bar{r}} \operatorname{Li}_{\bar{r}}(z)
$$

где $C_{\bar{r}}-$ некоторые челые неотрицательные числа. При этом младиий в лексикографическом упорядочении индексов обобщенных полилогарифмов, присутствующ, их с ненулевыми коэффиииентами в сумме справа, имеет вид $\left(\{1\}_{j_{1}},\{2\}_{j_{2}}, \ldots\right.$, $\{k\}_{j_{k}}$ ) и соответствующий ему коэффициент $C_{\bar{r}}$ ненулевой. 
ДоКАЗАТЕЛЬСТво проведем индукцией по $m=j_{1}+2 j_{2}+\cdots+k j_{k}$. При $m=1$ утверждение очевидно.

Пусть $m>1$ и для $m-1$ лемма уже доказана. Положим $n=j_{1}+\cdots+j_{k}$. Согласно (2)

$$
\begin{aligned}
\frac{d}{d z}\left(\mathrm{Li}_{1}^{j_{1}}(z) \cdots \mathrm{Li}_{k}^{j_{k}}(z)\right)= & \frac{j_{1}}{1-z} \mathrm{Li}_{1}^{j_{1}-1}(z) \mathrm{Li}_{2}^{j_{2}}(z) \cdots \mathrm{Li}_{k}^{j_{k}}(z) \\
& +j_{2} \frac{1}{z} \cdot \mathrm{Li}_{1}^{j_{1}+1}(z) \mathrm{Li}_{2}^{j_{2}-1}(z) \mathrm{Li}_{3}^{j_{3}}(z) \cdots \mathrm{Li}_{k}^{j_{k}}(z)+\cdots \\
& +j_{k} \frac{1}{z} \cdot \mathrm{Li}_{1}^{j_{1}}(z) \cdots \mathrm{Li}_{k-2}^{j_{k-2}}(z) \mathrm{Li}_{k-1}^{j_{k-1}+1}(z) \mathrm{Li}_{k}^{j_{k}-1}(z) \\
= & \frac{j_{1}}{1-z} \sum_{\substack{\mid \bar{r} \overline{\mid}=m-1 \\
l(\bar{r})=n-1}} C_{\bar{r}}^{(1)} \operatorname{Li}_{\bar{r}}(z)+\frac{1}{z} \sum_{s=2}^{k} j_{s} \sum_{\substack{|\bar{r}|=m-1 \\
l(\bar{r})=n}} C_{\bar{r}}^{(s)} \operatorname{Li}_{\bar{r}}(z)
\end{aligned}
$$

Интегрируя полученное равенство в соответствии с (2), мы приходим к требуемой формуле. При этом, очевидно, в случае $j_{1} \neq 0$ полилогарифм с самым младшим индексом получится интегрированием 'самого младшего' полилогариффма в сумме, стоящей при $j_{1}$, т.е. при интегрировании

$$
\operatorname{Li}_{\left(\{1\}_{j_{1}-1},\{2\}_{j_{2}}, \ldots,\{k\}_{j_{k}}\right)}(z),
$$

коэффициент при котором по индуктивному предположению ненулевой. После его интегрирования получим по условию леммы полилогарифм

$$
\operatorname{Li}_{\left(\{1\}_{j_{1}},\{2\}_{j_{2}}, \ldots,\{k\}_{j_{k}}\right)}(z)
$$

с ненулевым коэффициентом. Если же $j_{1}=0$, то обозначим через $s$ целое число такое, что $2 \leqslant s \leqslant k, j_{1}=\cdots=j_{s-1}=0, j_{s} \neq 0$. Рассмотрим самые младшие индексы полилогарифмов в суммах, стояших соответственно при $j_{s}, j_{s+1}, \ldots, j_{k}$. Из них младше других будет индекс полилогарифма

$$
\operatorname{Li}_{\left(s-1,\{s\}_{j_{s}-1},\{s+1\}_{j_{s+1}}, \ldots,\{k\}_{j_{k}}\right)}(z)
$$

входящего в сумму при $j_{s}$. Как и в случае $j_{1} \neq 0$, соответствуюший ему коэффициент ненулевой по индуктивному предположению. Интегрируя, получим полилогарифмм

$$
\operatorname{Li}_{\left(\{s\}_{j_{s}},\{s+1\}_{j_{s+1}}, \ldots,\{k\}_{j_{k}}\right)}(z)
$$

также с ненулевым коэффициентом, индекс которого опять-таки удовлетворяет формулировке леммы. Таким образом, лемма доказана.

ТЕОРема 4. Для любого натурального числа $k$ функиии $\operatorname{Li}_{1}(z), \ldots, \operatorname{Li}_{k}(z)$ алгебраически независимы над $\mathbb{C}(z)$. 
ДокАЗАтЕЛЬСтво. Пусть $A\left(x_{1}, \ldots, x_{k}\right) \in \mathbb{C}(z)\left[x_{1}, \ldots, x_{k}\right], A \not \equiv 0$ и $A\left(\operatorname{Li}_{1}(z), \ldots\right.$, $\left.\mathrm{Li}_{k}(z)\right)=0$. Рассмотрим мономы, входящие в $A$. Выберем из них старшие по степени по совокупности переменных, из которых возьмем старший в лексикографическом упорядочении. Полученньй моном мы назовем старшим. Пусть он имеет вид $\alpha x_{1}^{j_{1}} \cdots x_{k}^{j_{k}}$, где $\alpha$ - ненулевая рациональная функция.

Согласно лемме 2

$$
A\left(\operatorname{Li}_{1}(z), \ldots, \operatorname{Li}_{k}(z)\right)=\sum_{\bar{p}} C_{\bar{p}} \operatorname{Li}_{\bar{p}}(z),
$$

где $C_{\bar{p}} \in \mathbb{C}(z)$, причем при подстановке $\operatorname{Li}_{1}(z), \ldots, \operatorname{Li}_{k}(z)$ в $\alpha x_{1}^{j_{1}} \cdots x_{k}^{j_{k}}$ и представлении произведения с помошью леммы 2 помимо прочих слагаемых появится полилогарифм с индексом $\bar{s}=\left(\{1\}_{j_{1}},\{2\}_{j_{2}}, \ldots\{k\}_{j_{k}}\right)$, коэффициент при котором будет равен $C \alpha$, где $C-$ натуральное число. Этот набор будет младше индекса $\bar{p}$ любого другого обобщенного полилогарифма из (11) для которого вьполнено $l(\bar{p})=j_{1}+j_{2}+\cdots+j_{k}$, что следует из выбора старшего монома и леммы 2 . Таким образом, $C_{\bar{s}}=C \alpha \not \equiv 0$, что противоречит теореме 3 . Полученное противоречие завершает доказательство теоремы.

\section{СПИСОК ЦИТИРОВАННОЙ ЛИТЕРАТУРЫ}

[1] Minh Hoang Ngoc, Petitot M., Van Der Hoeven J. L'algèbre des polylogarithmes par les séries génératrices // Séries formelles et combinatoire algébrique. Barcelone, 1999.

[2] Сорокин В.Н. О мере трансцендентности числа $\pi^{2}$ // Матем. сб. 1996. Т. 187. № 12. C. $87-121$.

[3] Hoffman M. E. Multiple harmonic series // Pacific J. Math. 1992. V. 152. № 2. P. 275-290.

[4] Minh Hoang Ngoc, Petitot M. Lyndon words, polylogarythms and Riemann's $\zeta$ function // Discrete Math. 2000. V. 217. №1-3. P. 273-292.

[5] Minh Hoang Ngoc, Petitot M., Van Der Hoeven J. Shuffle algebra and polylogarithms // Discrete Math. 2000. V. 225. № 1-3. P. 217-230.

[6] Шидловский А. Б. О трансцендентности и алгебраической независимости значений некоторых E-функций // Вестн. МГУ. Сер. 1. Матем., мех. 1961. № 5. С. 44-59.

[7] Зудилин В. В. О мере иррациональности значений $G$-функций // Изв. РАН. Сер. матем. 1996. Т. 60 . № 1. С. $87-114$.

Московский государственный университет им. М.В. Ломоносова

Поступило

19.02 .2002

Исправленный вариант

24.07.2002 